\title{
Exercise training response heterogeneity: physiological and molecular insights
}

\author{
Lauren M. Sparks ${ }^{1,2}$
}

Received: 19 June 2017 / Accepted: 18 August 2017 /Published online: 14 October 2017

(C) Springer-Verlag GmbH Germany 2017

\begin{abstract}
The overall beneficial effects of exercise are well studied, but why some people do not respond favourably to exercise is less understood. The National Institutes of Health Common Fund has recently launched the large-scale discovery project 'Molecular Transducers of Physical Activity in Humans' to examine the physiological and molecular (i.e. genetic, epigenetic, lipidomic, metabolomic, proteomic, etc.) responses to exercise training. A nationwide, multicentre clinical trial such as this one also provides a unique opportunity to robustly investigate the non-response to exercise in thousands of individuals that have undergone supervised aerobic- and resistance-based exercise training interventions. The term 'non-responder' is used here to address the lack of a response (to an exercise intervention) in an outcome specified a priori. Cardiorespiratory fitness $\left(\mathrm{VO}_{2 \text { peak }}\right)$ as an exercise response variable was recently reviewed; thus, this review focuses on metabolic aspects of the non-response to exercise training. Integrated -omics platforms are discussed as an approach to disentangle the complicated relationships between endogenous and exogenous factors that drive the lack of a response to exercise in some individuals. Harnessing the power of combined -omics platforms with deep clinical phenotyping of human study participants will advance the field of exercise metabolism and shift the paradigm, allowing exercise
\end{abstract}

Electronic supplementary material The online version of this article (https://doi.org/10.1007/s00125-017-4461-6) contains a slide of the figure for download, which is available to authorised users.

Lauren M. Sparks

lauren.sparks@ flhosp.org

1 Translational Research Institute for Metabolism and Diabetes, Florida Hospital, 301 E Princeton Street, Orlando, FL 32804, USA

2 Sanford Burnham Prebys Medical Discovery Institute, Orlando, FL, USA interventions to be targeted at those most likely to benefit and identifying novel approaches to treat those who do not.

Keywords Diabetes $\cdot$ Epigenetics $\cdot$ Exercise $\cdot$

Heterogeneity $\cdot$ Human $\cdot$ Metabolism $\cdot$ Muscle $\cdot$ Response $\cdot$ Review $\cdot$ Training

\begin{abstract}
Abbreviations
NIH National Institutes of Health

STRRIDE Studies Targeting Risk Reduction Interventions through Defined Exercise
\end{abstract}

\section{What is a non-response?}

From a statistical perspective, a non-response is the lack of a difference between a control and a treatment condition with respect to a specific variable. A physiological non-response, however, is likely driven by genetic code and the mechanisms by which it is transcribed, translated and post-translationally modified. Drug resistance is an archetype of non-response that links physiology with genetics. For example, some individuals are 'rapid' or 'slow' metabolisers of a prescribed drug based solely on their genetic predispositions, and simple DNA tests can now identify these at-risk populations (reviewed in [1]). Combination drug therapy for initial non-response, acquired resistance or adverse response is usually the next course of action; therefore, tailoring treatment to the genetic code is a viable strategy for some diseases and conditions.

Response heterogeneity is not unique to pharmaceutical therapies; lifestyle interventions, such as dietary weight loss and exercise, have also been linked to physiological, genetic and epigenetic factors. Differences in energy efficiency are important physiological regulators of body weight and 
weight-loss success (reviewed in [2]). A National Institutes of Health (NIH) working group recently published a report on the use of genomic information to guide weight management, thus enabling precision prescription for weight loss [3]. Thus, use of (epi)genetics to prescribe tailored exercise interventions could be a worthwhile treatment approach for individuals previously identified as 'non-responders' to exercise.

\section{How should we evaluate the non-response to exercise?}

\section{Selecting an outcome response variable}

A physiological non-response to exercise in one outcome does not equal a non-response in all outcomes. An individual may reap benefits in response to an exercise intervention other than the chosen response variable. For instance, a person may experience a reduction in blood pressure but no reduction in fasting glucose. Which one is more important? It depends on the current health status of that person and their health-related goals. An exercise non-responder for their relevant outcome could seek supplemental treatment options (e.g. diet, pharmacotherapy) in addition to exercise, and the supplemental therapy could have an additive effect when combined with the exercise such that the relevant outcome is now positively affected. Resolution of metabolic perturbations through combinations of lifestyle and pharmaceutical interventions emphasises the clinical relevance of identifying and studying non-responsiveness to exercise.

In a cohort of 161 individuals with type 2 diabetes who underwent 9 months of supervised exercise training [4], 43\% showed no improvement in cardiorespiratory fitness and were deemed 'fitness non-responders'; however, both fitness nonresponders and fitness responders showed significant improvements in $\mathrm{HbA}_{1 \mathrm{c}}$, waist circumference and body-fat per cent [5]. Therefore, it was not necessary to improve cardiorespiratory fitness to improve glycaemic control, which is arguably a more important outcome in a population with type 2 diabetes. Similarly, in a recent report from the Studies Targeting Risk Reduction Interventions through Defined Exercise (STRRIDE) trial, aerobic training increased $V \mathrm{O}_{2 \text { peak }}$ and insulin sensitivity but had no effect on beta cell function [6]. While the participants in STRRIDE were healthy overall, some had impaired fasting glucose. Thus, an exercise intervention that improved multiple aspects of glucose homeostasis and insulin secretion (rather than just one) would likely be a better target treatment for those individuals with impaired fasting glucose.

\section{Does non-response to exercise actually exist?}

A few recent reports have refuted the notion of nonresponders to exercise, stating that to convert a nonresponder into a responder it is simply a matter of changing the volume, type or intensity of the training regimen. In one report, the investigators found that $\dot{V} \mathrm{O}_{2 \text { peak }}$ non-response was abolished after increasing the exercise dose in healthy individuals [7]. While these data are strong in terms of $\dot{V} \mathrm{O}_{2 \text { peak }}$ as the response variable, other important response variables known to be improved by exercise training were not assessed (e.g. glucose homeostasis, insulin secretion, blood pressure). One can only speculate as to whether these 'responders' may still have been considered responders if other health-related outcomes had been evaluated. Another report concluded that non-responsiveness to months of resistance training was not apparent since every participant experienced a positive adaptive response in at least one training outcome [8]. In this case, the term 'non-responder' is more about semantics since the authors demonstrate a non-response in some of the chosen response variables (e.g. muscle fibre size, lean body mass, leg strength) across participants. Even the authors of reports that refute the so-called 'myth' of exercise non-response might agree that the term 'non-responder' depends solely on the chosen clinical outcome, and that a non-responder in one outcome may not be a non-responder in another outcome. As technology advances and our understanding of the mechanisms driving exercise responses improves, scientists can continue to narrow the focus on clinical outcomes that are critical to improving the health of an individual and healthcare practitioners can prescribe exercise individually, rather than broadly prescribing the same exercise training regimens to all.

A thought-provoking paper has also addressed this issue of determining the outcome variable by which non-responders and responders are classified from a statistical perspective [9]. The report used data from a previously published study on responders and non-responders and determined that 'if uncertainty of classification is ignored during the analysis, then statistical inference may be unreliable', and that hierarchical modelling facilitates the correct modelling of the latent variable in terms of predictor variables and hypothesised biological relationships [9]. Ergo, not only is the choice of response variable critical but also how this variable is statistically evaluated to classify non-responders is vitally important to the downstream biological interpretations.

\section{Endogenous factors as drivers of non-response to exercise}

\section{Age and sex}

From a disease perspective, endogenous means 'not attributable to any external or environmental factor'. Ageing is associated with impaired hypertrophic responses to resistance exercise training (anabolic resistance) [10-14]. However, equal adaptations to high-intensity aerobic training in insulin sensitivity, $\dot{V} \mathrm{O}_{2 \text { peak }}$ and skeletal muscle mitochondrial respiration 
have been reported in older and younger individuals [15], and chronically trained older individuals have high mitochondrial content, function and exercise efficiencies [16]. While age is an important endogenous factor when considering exercise non-response, the type and duration of exercise may equally matter in the context of ageing. Recent studies report no sexspecific differences in the expression of substrate metabolism genes and fibre type following acute bouts of exercise and short-term interventions [8, 17], although one study reported that women exhibited a significantly greater magnitude of exercise-induced upregulation in proteins related to muscle metabolism [18]. As such, the influence of sex on adaptive responses of skeletal muscle metabolism to exercise continues to be debated.

\section{Duration of diabetes, family history of diabetes and blood glucose levels}

In one study, a longer duration of diabetes was associated with a blunted improvement in $\mathrm{HbA}_{1 \mathrm{c}}$ following 9 months of exercise [19], suggesting that intervening with exercise earlier, rather than later, in the disease progression yields greater chances of a positive exercise response, at least in terms of glycaemic control. Interestingly, exercise training has been shown to elicit more profound improvement in insulin sensitivity in female offspring of individuals with type 2 diabetes (i.e. family history positive) than in those without a family history of diabetes [20]. In contrast, relatives of individuals with type 2 diabetes did not experience any increase in the rate of skeletal muscle ATP synthesis following three bouts of aerobic exercise when compared with healthy individuals [21]. In a similar cohort of individuals at high risk for developing type 2 diabetes (family history positive or previous gestational diabetes), non-responders showed no improvements in insulin sensitivity following 8 weeks of highintensity aerobic training. The non-responders also displayed increased transcript levels of $T G F-\beta 1$ (also known as TGFB1) and its target genes and an overall suppression of mitochondrial regulators in their muscle [22].

Ambient hyperglycaemia can blunt the metabolic exercise response [23]. For every $1 \mathrm{mmol} / \mathrm{l}$ increase in pre-training $2 \mathrm{~h}$ blood glucose levels above $13.1 \mathrm{mmol} / 1$, there was a $0.2 \mathrm{mmol} / \mathrm{l}$ loss of improvement in $2 \mathrm{~h}$ blood glucose levels following 12-16 weeks of aerobic training in individuals with impaired glucose tolerance or type 2 diabetes [24]. STRRIDE demonstrated a significant inverse correlation between baseline fasting glucose and the change in insulin sensitivity following 8 months of aerobic training in overweight individuals [6]. Participants with normal fasting glucose experienced an improvement in insulin sensitivity but participants with impaired fasting glucose experienced a decrease in insulin sensitivity with aerobic training. Clearly, blood glucose levels (whether influenced by genetic or environmental factors) are closely linked with metabolic non-responses to exercise training. Future studies aimed at resolving hyperglycaemia through either pharmacological or dietary intervention prior to introducing an exercise regimen may be an effective strategy for targeting these non-responders and improving their chances of success.

\section{Molecular predictors of the exercise response}

The mechanisms by which exercise improves health outcomes are poorly understood. In 2014, the NIH convened a workshop to identify major gaps in knowledge and to formulate potential strategies for catalysing progress in the field. It subsequently launched the large-scale discovery project 'Molecular Transducers of Physical Activity in Humans' to aid the understanding of physiological and molecular responses to exercise training. Identification of the mechanisms that underlie the link between exercise and improved health holds extraordinary promise for the discovery of novel therapeutic targets and development of precision exercise medicine.

Genetics Classic genetic studies identified a heredity component to the exercise training response, particularly for $\dot{V} \mathrm{O}_{2 \text { peak }}$. More recent findings have revealed genetic factors associated with the metabolic response to exercise, whereby individuals within a family respond more similarly than those from different families [25-27]. In these studies, genome-wide linkage scans were performed in $>400$ individuals at baseline and following a 20 week aerobic training intervention. Researchers found specific quantitative trait loci linked with changes in plasma insulin [28], triacylglycerol levels [29] and glucose homeostasis [30] in response to exercise. This suggests that sequence variations dictate exercise response. The field continues to expand and includes the influences of genetics and epigenetics, in addition to physiological and lifestyle factors, on the response (or lack of) to acute and chronic exercise.

Epigenetics A single muscle contraction is sufficient to induce hypomethylation of the promoter regions of key substrate metabolism genes, resulting in upregulated transcription and subsequent protein translation and action [31]. It is tempting to speculate that non-responders to an exercise intervention have hypermethylated promoter regions of key substrate metabolism genes in response to a contraction and that this contributes to the lack of improvement in certain metabolic outcomes. While cause and consequence of exercise-induced changes in insulin sensitivity and muscle mitochondrial function have not been firmly established, these two clinical outcomes have frequently been associated. 
Transcriptomics We recently demonstrated that $\sim 20 \%$ of individuals with type 2 diabetes did not show improvements in $\mathrm{HbA}_{1 \mathrm{c}}$, muscle mitochondrial content, body fat or BMI following 9 months of exercise, and that these nonresponders had a distinct basal muscle transcriptional profile of genes related to substrate metabolism [32]. Identifying predictors of a non-response, whether genetic, epigenetic or metabolic, prior to an exercise intervention could pave the way for targeted exercise interventions and prescriptions.

Metabolomics A recent study identified metabolite signatures of exercise training in human skeletal muscle that were related to mitochondrial remodelling and cardiometabolic fitness [33]. Participants showing no improvement in insulin sensitivity following 6 months of aerobic and/or resistance training also showed no improvement in certain aspects of their muscle metabolite profiles. While these metabolite and transcriptional signatures were not basal predictors of a non-response, but rather changes in response to the exercise, this study integrated two -omics datasets highlighting new avenues for mechanistic research aimed at understanding the health benefits of exercise and the lack of a response by some individuals.

\section{Exogenous factors also influence non-response to exercise}

\section{Duration, intensity and type of exercise training}

From a biological perspective, exogenous means 'relating to or developing from external factors'. Three primary exogenous factors can contribute to the exercise non-responder phenotype: (1) duration/volume/dose of training; (2) intensity of training; and (3) type/mode of training. In individuals with type 2 diabetes who participated in either a low-intensity/ long-duration or a high-intensity/short-duration training regimen, the persistent training-induced improvements in insulin sensitivity depended more on training duration than intensity, with the same level of energy expenditure in both groups [34]. As such, a non-responder in the high-intensity/short-duration group might have been a responder in the low-intensity/longduration group. A randomised, controlled trial in postmenopausal overweight/obese women revealed that women who were younger, less fit or exercised more during the trial had greater odds of improving their $\dot{V} \mathrm{O}_{2 \text { peak }}$ with training. The most important finding of this study was that a greater volume of exercise was associated with a lower probability of being a non-responder [35]. Other reports have demonstrated a doseresponse relationship between exercise and reductions in blood pressure [36] and inflammation [37] in these same postmenopausal women, with the highest dose being the most effective (up to $200 \mathrm{~min} /$ week, which is $50 \mathrm{~min}$ longer than the dose currently recommended in the USA for producing substantial health benefits according to physical activity guidelines [38]). In agreement, a recent report determined that response to a dose eliminates non-response in $\dot{V} \mathrm{O}_{2 \text { peak }}$ of healthy individuals [7]. Such a high volume of exercise training, however, may not be feasible or practical for all individuals.

Intense intermittent exercise has become increasingly recognised as a powerful stimulus to induce many of the physiological adaptations typically associated with traditional moderate-intensity continuous exercise training but in a fraction of the time. A recent study in young active men demonstrated that single-leg cycling performed in an interval vs a continuous manner elicited superior mitochondrial adaptations in the skeletal muscle despite equal total work [39, 40], although response heterogeneity was evident. Beneficial effects on insulin sensitivity have also been reported following short durations of training in healthy young men [41]. However, fasting plasma insulin and glucose concentrations remained unchanged (i.e. the men were considered non-responsive), which again raises the issue of the clinical outcome and what is most relevant for an individual's health. Whether high-intensity interval training is advantageous and to which subpopulation it is best suited remains to be determined.

\section{Can individual responses to exercise be overcome by different training regimens?}

Given the fact that aerobic training induces different signalling pathways to those induced by resistance training (reviewed in [42]), each person will likely respond differently to a particular type of training. In one cohort of healthy young men and women, individuals were segregated into three groups-'extreme responders', 'modest responders' and 'nonresponders'-based on differential magnitudes of myofibre hypertrophy following a progressive resistance training programme [43]. This begs the question of whether these nonresponders to resistance training might have shown a better response to aerobic training. A recent study reported that a combination of low-volume/vigorous-intensity combined aerobic and resistance training was the most favourable in terms of improvement in insulin sensitivity in overweight adults [33]. Combined aerobic and resistance training is most beneficial for glucose homeostasis in individuals with type 2 diabetes $[4,44]$, yet some of these individuals are still classified as non-responders even to combined training [32]. By changing training modalities, significantly greater clinical benefits can potentially be obtained in terms of muscle quality, glycaemic control and insulin sensitivity. Adjuvant therapy might be a possible solution; combining lower doses and differing intensities of exercise training with other lifestyle modifications (e.g. diet) or medications could enhance the exercise response in people previously identified as non-responders. 


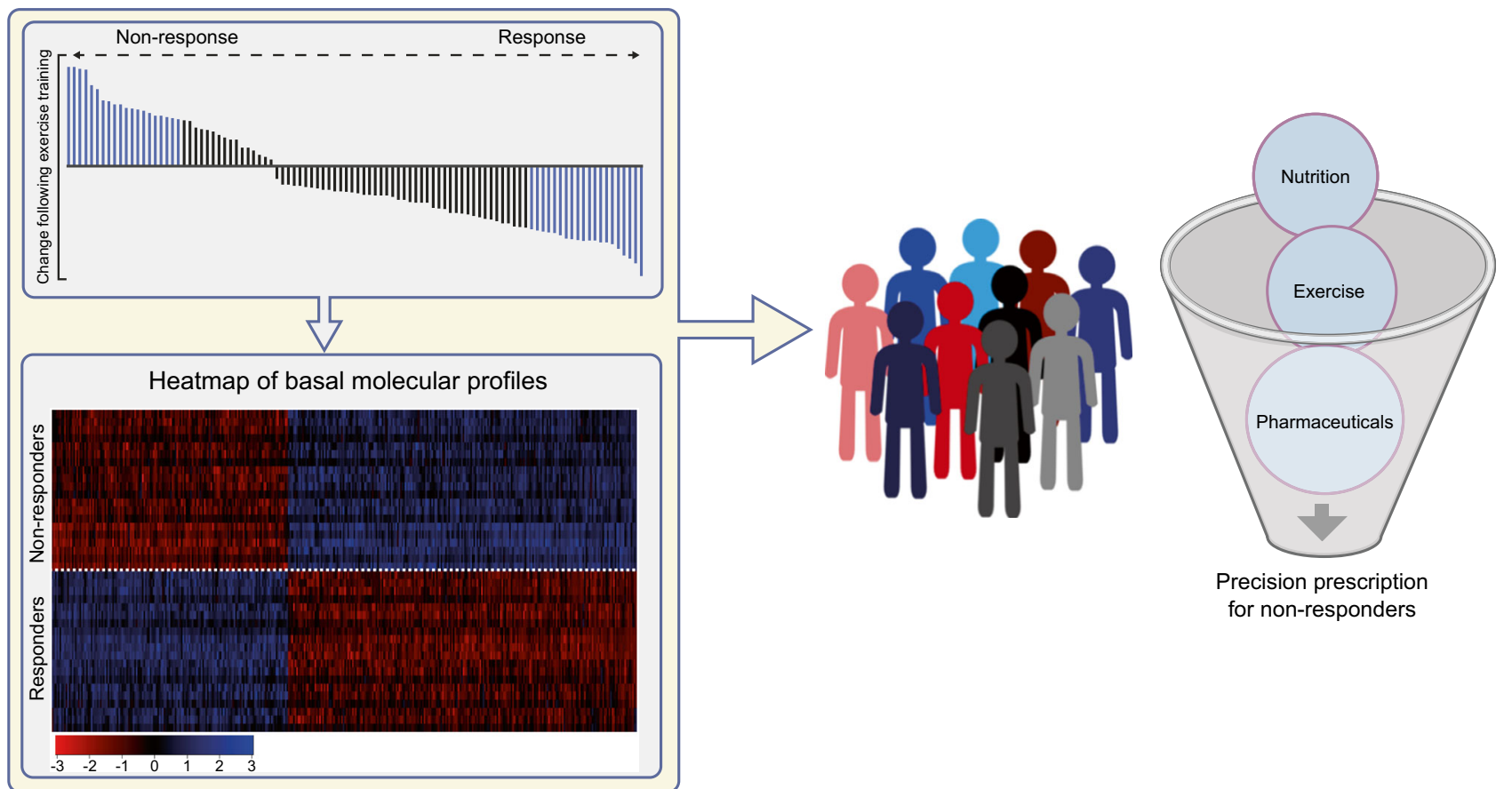

Fig. 1 A proposal for using both clinical and molecular data to identify non-responders at baseline and devise an intervention tailored to suit their individual needs. For any given response variable, a change following an exercise training intervention can be evaluated per individual participant. In this example waterfall plot, each bar represents an individual's response, with responses in the upper and lower $20 \%$ (based on previously published data from our group demonstrating that $20 \%$ of individuals with type 2 diabetes did not respond favourably to exercise training in terms of glycaemic control $[32,52])$ highlighted in blue. The directions of the hypothetical response and non-response are indicated by dashed arrows. Using the upper and lower $20 \%$ of responses, basal molecular

\section{Other factors to consider when evaluating exercise non-response}

Dietary intake has been touted as a critical regulator of the exercise response, especially in the context of the feast/ famine and activity/rest cycle, and has therefore been rigorously reviewed $[45,46]$. Related to dietary intake, a potential endogenous predictor of the non-response to exercise is an individual's gut microbiome. The gut microbiome and its influence on host behaviour, intestinal barrier and immune function are believed to be a critical aspect of the brain-gut axis [47]. While the influence of the gut microbiome on exercise non-response is currently untested in humans, recent evidence in murine models shows that there is a strong correlation between physical and emotional stress during exercise and changes in gastrointestinal microbiota composition [48]. This is an exciting field, ripe with opportunities for investigating the influence of the gut microbiome on the exercise non-response.

The time of day at which exercise training is performed, and medication use are other exogenous factors related to nonresponse. One study found that $60 \mathrm{~min}$ of aerobic exercise increased $24 \mathrm{~h}$ whole-body fat oxidation only when it was profiles can be generated to distinguish the responders and non-responders at baseline and visualised via hierarchical clustering in a heatmap. By combining deep clinical phenotyping of the exercise-training response with basal molecular profiles, personalised prescriptions using exercise training (alone or in combination with nutritional and pharmaceutical regimens also based on an individual's clinical and molecular profiles) can be precisely implemented to maximise each person's success. The values in the waterfall plot were simulated for illustration purposes. The simulated data in the heatmap were generated from a masking microarray 'hit-list' dataset

performed before breakfast in the post-absorptive state [49]. More people are taking medications to combat the adverse effects of obesity, impaired glucose tolerance and related metabolic diseases. Biguanides (e.g. metformin) have been shown to blunt the metabolic response to exercise and are classified as ATP synthase inhibitors [50]. Prospective studies in medication-naive individuals with impaired glucose tolerance have demonstrated a blunted exercise-induced improvement in insulin sensitivity when metformin was included in the treatment regimen [51]. Hence, exogenous factors should be considered when prescribing exercise interventions.

\section{Concluding remarks}

Non-responders to exercise exist. As many as $20 \%$ of individuals with type 2 diabetes do not respond favourably to exercise training in terms of glycaemic control and muscle mitochondrial function $[32,52]$. Non-response to exercise, however, is not unique to diseased or untrained populations, emphasising the importance of selecting a response variable 


\section{Summary}

Exercise response heterogeneity exists and a non-response in one outcome does not equal a non-response in all outcomes. The following points are important when evaluating exercise response:

Identify the response variable a priori Consider the response variable within the context of anticipated health-related outcomes of the exercise intervention when generating the selection criteria

2 Technical precision Ensure technical precision for the measurement of the response variable to avoid false discoveries

3 Endogenous factors Consider the influence of endogenous factors that are inherent to the individual on exercise response (e.g. age, sex, race, (epi)genetics, gut microbiome, etc.)

4 Exogenous factors Consider the influence of exogenous factors that are within the individual's control on exercise response (e.g. mode, intensity, duration, time of day, nutritional status, etc.)

5 Molecular mechanisms and predictors of response Downstream interrogations of the mechanism(s) driving response heterogeneity can be focused on single pathways and molecules but should be validated in other data sets and optimally integrated with multiple measures of molecular transducers (e.g. metabolites, lipids, proteins, genes, etc.)

a priori. Once a response variable has been established, analytical rigour is paramount. The statistical power of the chosen outcome should be assessed prior to classifying the exercise response of participants in a study, thus ensuring that even the subtlest differences in a biologically meaningful outcome can be detected with confidence and investigated more thoroughly to determine underlying mechanisms. Both endogenous factors (inherent to the individual and, potentially, a predictor of the response) and exogenous factors (environmental and manipulated by the individual) contribute to exercise response heterogeneity and can be exploited to achieve maximal beneficial responses on an individual basis. Pharmaceutical therapies and dietary weight-loss regimens have embraced this concept of non-response to treatment and are well on their way to leveraging an individual's genomic and/or epigenomic profile to tailor their treatments. As the exercise metabolism field continues to combine the plethora of -omics data with deep clinical phenotyping of study participants in clinical exercise trials, we will move closer towards shifting the paradigm by allowing exercise prescriptions to be targeted at those most likely to benefit and identifying novel approaches to treat those who do not (Fig. 1).

Acknowledgements Sincere gratitude is expressed to S. R. Smith (Translational Research Institute, USA) and T. S. Church (ACAP Health Consulting, USA) who have profoundly shaped the author's thoughts about response heterogeneity to both exercise and weight loss over the past 15 years. B. H. Goodpaster (Translational Research Institute, USA) is also thanked for devoting his time to thoughtful discussions about this topic, as well as the many others who have contributed to the studies and concepts highlighted in this review. Thanks is also given to $\mathrm{F}$. Yi for his help with Fig. 1.

Funding The author is supported by a grant from the American Diabetes Association (no. 7-13-JF-53).

Duality of interest The author declares that there is no duality of interest associated with this manuscript.

Contribution of statement The author was the sole contributor to this paper.

\section{References}

1. Pereira NL, Weinshilboum RM (2009) Cardiovascular pharmacogenomics and individualized drug therapy. Nat Rev Cardiol 6:632-638

2. Thrush AB, Dent R, McPherson R, Harper ME (2013) Implications of mitochondrial uncoupling in skeletal muscle in the development and treatment of obesity. FEBS J 280:5015-5029

3. Bray MS, Loos RJ, McCaffery JM et al (2016) NIH working group report-using genomic information to guide weight management: from universal to precision treatment. Obesity 24:14-22

4. Church TS, Blair SN, Cocreham S et al (2010) Effects of aerobic and resistance training on hemoglobin A1c levels in patients with type 2 diabetes: a randomized controlled trial. JAMA 304:22532262

5. Pandey A, Swift DL, McGuire DK et al (2015) Metabolic effects of exercise training among fitness-nonresponsive patients with type 2 diabetes: the HART-D study. Diabetes Care 38:1494-1501

6. AbouAssi H, Slentz CA, Mikus CR et al (2015) The effects of aerobic, resistance, and combination training on insulin sensitivity and secretion in overweight adults from STRRIDE AT/RT: a randomized trial. J Appl Physiol 118:1474-1482

7. Montero D, Lundby C (2017) Refuting the myth of non-response to exercise training: 'non-responders' do respond to higher dose of training. J Physiol 595:3377-3387

8. Churchward-Venne TA, Tieland M, Verdijk LB et al (2015) There are no nonresponders to resistance-type exercise training in older men and women. J Am Med Dir Assoc 16:400-411

9. Barker RJ, Schofield MR (2008) Classifying individuals as physiological responders using hierarchical modeling. J Appl Physiol 105:555-560

10. Brook MS, Wilkinson DJ, Mitchell WK et al (2016) Synchronous deficits in cumulative muscle protein synthesis and ribosomal biogenesis underlie age-related anabolic resistance to exercise in humans. J Physiol 594:7399-7417 
11. Welle S, Totterman S, Thornton C (1996) Effect of age on muscle hypertrophy induced by resistance training. J Gerontol A Biol Sci Med Sci 51:M270-M275

12. Kosek DJ, Kim JS, Petrella JK, Cross JM, Bamman MM (2006) Efficacy of 3 days/wk resistance training on myofiber hypertrophy and myogenic mechanisms in young vs. older adults. J Appl Physiol 101:531-544

13. Greig CA, Gray C, Rankin D et al (2011) Blunting of adaptive responses to resistance exercise training in women over $75 \mathrm{y}$. Exp Gerontol 46:884-890

14. Mero AA, Hulmi JJ, Salmijarvi H et al (2013) Resistance training induced increase in muscle fiber size in young and older men. Eur $\mathrm{J}$ Appl Physiol 113:641-650

15. Robinson MM, Dasari S, Konopka AR et al (2017) Enhanced protein translation underlies improved metabolic and physical adaptations to different exercise training modes in young and old humans. Cell Metab 25:581-592

16. Broskey NT, Boss A, Fares EJ et al (2015) Exercise efficiency relates with mitochondrial content and function in older adults. Phys Rep 3:e12418

17. Skelly LE, Gillen JB, MacInnis MJ et al (2017) Effect of sex on the acute skeletal muscle response to sprint interval exercise. Exp Physiol 102:354-365

18. Peters SJ, Samjoo IA, Devries MC, Stevic I, Robertshaw HA, Tarnopolsky MA (2012) Perilipin family (PLIN) proteins in human skeletal muscle: the effect of sex, obesity, and endurance training. Appl Physiol Nutr Metab 37:724-735

19. Johannsen NM, Sparks LM, Zhang Z et al (2013) Determinants of the changes in glycemic control with exercise training in type 2 diabetes: a randomized trial. PLoS One e62973:8

20. Barwell ND, Malkova D, Moran CN et al (2008) Exercise training has greater effects on insulin sensitivity in daughters of patients with type 2 diabetes than in women with no family history of diabetes. Diabetologia 51:1912-1919

21. Kacerovsky-Bielesz G, Chmelik M, Ling C et al (2009) Short-term exercise training does not stimulate skeletal muscle ATP synthesis in relatives of humans with type 2 diabetes. Diabetes 58:1333-1341

22. Bohm A, Hoffmann C, Irmler M et al (2016) TGF- $\beta$ contributes to impaired exercise response by suppression of mitochondrial key regulators in skeletal muscle. Diabetes 65:2849-2861

23. Malin SK, Kirwan JP (2012) Fasting hyperglycaemia blunts the reversal of impaired glucose tolerance after exercise training in obese older adults. Diabetes Obes Metab 14:835-841

24. Solomon TP, Malin SK, Karstoft K, Haus JM, Kirwan JP (2013) The influence of hyperglycemia on the therapeutic effect of exercise on glycemic control in patients with type 2 diabetes mellitus. JAMA Intern Med 173:1834-1836

25. An P, Teran-Garcia M, Rice T et al (2005) Genome-wide linkage scans for prediabetes phenotypes in response to 20 weeks of endurance exercise training in non-diabetic whites and blacks: the HERITAGE Family Study. Diabetologia 48:1142-1149

26. Boule NG, Weisnagel SJ, Lakka TA et al (2005) Effects of exercise training on glucose homeostasis: the HERITAGE Family Study. Diabetes Care 28:108-114

27. Lakka TA, Lakka HM, Rankinen T et al (2005) Effect of exercise training on plasma levels of C-reactive protein in healthy adults: the HERITAGE Family Study. Eur Heart J 26:2018-2025

28. Lakka TA, Rankinen T, Weisnagel SJ et al (2003) A quantitative trait locus on $7 \mathrm{q} 31$ for the changes in plasma insulin in response to exercise training: the HERITAGE Family Study. Diabetes 52: $1583-1587$

29. Feitosa MF, Rice T, Rankinen T et al (2005) Evidence of QTLs on chromosomes $13 q$ and $14 q$ for triglycerides before and after 20 weeks of exercise training: the HERITAGE Family Study. Atherosclerosis 182:349-360
30. Ruchat SM, Rankinen T, Weisnagel SJ et al (2010) Improvements in glucose homeostasis in response to regular exercise are influenced by the PPARG Pro12Ala variant: results from the HERITAGE Family Study. Diabetologia 53:679-689

31. Barres R, Yan J, Egan B et al (2012) Acute exercise remodels promoter methylation in human skeletal muscle. Cell Metab 15: 405-411

32. Stephens NA, Xie H, Johannsen NM, Church TS, Smith SR, Sparks LM (2015) A transcriptional signature of exercise resistance in skeletal muscle of individuals with type 2 diabetes mellitus. Metabolism 64:999-1004

33. Huffman KM, Koves TR, Hubal MJ et al (2014) Metabolite signatures of exercise training in human skeletal muscle relate to mitochondrial remodelling and cardiometabolic fitness. Diabetologia 57:2282-2295

34. Li J, Zhang W, Guo Q et al (2012) Duration of exercise as a key determinant of improvement in insulin sensitivity in type 2 diabetes patients. Tohoku J Exp Med 227:289-296

35. Sisson SB, Katzmarzyk PT, Earnest CP, Bouchard C, Blair SN, Church TS (2009) Volume of exercise and fitness nonresponse in sedentary, postmenopausal women. Med Sci Sports Exerc 41:539 545

36. Church TS, Earnest CP, Skinner JS, Blair SN (2007) Effects of different doses of physical activity on cardiorespiratory fitness among sedentary, overweight or obese postmenopausal women with elevated blood pressure: a randomized controlled trial. JAMA 297:2081-2091

37. Johannsen NM, Swift DL, Johnson WD et al (2012) Effect of different doses of aerobic exercise on total white blood cell (WBC) and WBC subfraction number in postmenopausal women: results from DREW. PLoS One 7:e31319

38. Office of Disease Prevention and Health Promotion (2015) Dietary guidelines for Americans 2015-2020. Available from https://health. gov/dietaryguidelines/2015/guidelines/appendix-1/. Accessed 25 September 2017

39. MacInnis MJ, Gibala MJ (2017) Physiological adaptations to interval training and the role of exercise intensity. J Physiol 595:29152930

40. MacInnis MJ, Zacharewicz E, Martin BJ et al (2017) Superior mitochondrial adaptations in human skeletal muscle after interval compared to continuous single-leg cycling matched for total work. J Physiol 595:2955-2968

41. Babraj JA, Vollaard NB, Keast C, Guppy FM, Cottrell G, Timmons JA (2009) Extremely short duration high intensity interval training substantially improves insulin action in young healthy males. BMC Endocr Disord 9:3

42. Pesta DH, Goncalves RLS, Madiraju AK, Strasser B, Sparks LM (2017) Resistance training to improve type 2 diabetes: working toward a prescription for the future. Nutr Metab 14:24

43. Thalacker-Mercer A, Stec M, Cui X, Cross J, Windham S, Bamman M (2013) Cluster analysis reveals differential transcript profiles associated with resistance training-induced human skeletal muscle hypertrophy. Physiol Genomics 45:499-507

44. Sigal RJ, Kenny GP, Boule NG et al (2007) Effects of aerobic training, resistance training, or both on glycemic control in type 2 diabetes: a randomized trial. Ann Intern Med 147:357-369

45. Chakravarthy MV, Booth FW (2004) Eating, exercise, and thrifty genotypes: connecting the dots toward an evolutionary understanding of modern chronic diseases. J Appl Physiol 96:3-10

46. Heilbronn LK (2010) Fasting during exercise for fitness during feasting? J Physiol 588:4613-4614

47. Clark A, Mach N (2016) Exercise-induced stress behavior, gutmicrobiota-brain axis and diet: a systematic review for athletes. $\mathrm{J}$ Int Soc Sports Nutr 13:43 
48. Allen JM, Berg Miller ME, Pence BD et al (2015) Voluntary and forced exercise differentially alters the gut microbiome in C57BL/ 6J mice. J Appl Physiol 118:1059-1066

49. Iwayama K, Kurihara R, Nabekura Y et al (2015) Exercise increases 24-h fat oxidation only when it is performed before breakfast. EBioMedicine 2:2003-2009

50. Bridges HR, Jones AJ, Pollak MN, Hirst J (2014) Effects of metformin and other biguanides on oxidative phosphorylation in mitochondria. Biochem J 462:475-487
51. Malin SK, Gerber R, Chipkin SR, Braun B (2012) Independent and combined effects of exercise training and metformin on insulin sensitivity in individuals with prediabetes. Diabetes Care 35:131136

52. Stephens NA, Sparks LM (2015) Resistance to the beneficial effects of exercise in type 2 diabetes: are some individuals programmed to fail? J Clin Endocrinol Metab 100:43-52 